\title{
Using MSD prevention for cultural change in mining: Queensland Government/Anglo Coal Industry partnership
}

\author{
Trudy Tilbury ${ }^{\mathrm{a},{ }^{*}}$ and Liz Sanderson ${ }^{\mathrm{b}}$ \\ ${ }^{a}$ DEEDI Mines, Mackay, Queensland, Australia \\ ${ }^{\mathrm{b}}$ AAMC, Brisbane, Queensland, Australia
}

\begin{abstract}
Queensland Mining has a strong focus on safety performance, but risk management of health, including Musculoskeletal Disorders (MSDs) continues to have a lower priority. The reliance on individual screening of workers and lower level approaches such as manual handling training is part of the coal mining 'culture'. Initiatives such as the New South Wales and Queensland Mining joint project to develop good practice guidance for mining has allowed for a more consistent message on participatory ergonomics and prevention of MSD. An evidence based practice approach, including the introduction of participatory ergonomics and safe design principles, was proposed to Anglo American Coal operations in Queensland. The project consisted of a skills analysis of current health personnel, design of a facilitated participatory ergonomics training program, site visits to identify good practice and champions, and a graduated mentoring program for health personnel. Early results demonstrate a number of sites are benefiting from site taskforces with a focus on positive performance outcomes.
\end{abstract}

Keywords: Musculoskeletal Disorders, Participatory Ergonomics, Industry partnerships, Cultural change

\section{Introduction}

Coal mining has historically been an industry with high levels of fatalities and a number of well publicised long term health effects. Although the industry has significantly reduced fatalities in Australia, the sector continues to maintain a very significant focus on safety hazards, particularly in underground coal operations. The growth in the coal industry in Queensland has seen the expansion of recruitment and a focus on functional capacity evaluations, and other pre-employment screening of workers, with little change in the actual design of the hazardous manual tasks being undertaken. A number of recent initiatives in both New South Wales and Queensland have aimed to improve awareness of the role of ergonomics in prevention of Musculoskeletal Disorders, as well as improving the culture of the coal industry.

\footnotetext{
*Corresponding author. E-mail: trudy.tilbury@deedi.qld.gov.au
}

\section{Regulatory approach in Queensland Coal}

The coal industry in Queensland is governed by specific legislation that has evolved over time, and is influenced by political forces as well as legislative changes. The potential for multiple fatalities in underground coal and historical industrial arrangements have resulted in a more prescriptive and less proactive approach in most aspects of health. Many health issues were not understood by the inspectorate meant to enforce the legislation, meaning that the current legislation $(1999,2001)$ is focused on an acceptance of lower level approaches to health issues such as noise, musculoskeletal disorders, whole-body and hand-arm vibration. There are a number of reasons for this widespread acceptance of lower level controls such as personal protective equipment (Noise) and pre-employment screening and manual handling training (MSD), but a lack of a regulatory focus and 
appropriate skills within the regulator were identified in previous reviews of the Mines Inspectorate.

\subsection{Changes in the approach to health hazards since 2006}

The introduction of both Occupational Hygiene and Ergonomics roles within the Queensland Mines Inspectorate in 2006 allowed for some improvement in awareness of health hazards, as well as the introduction of more targeted guidance to the mining industry. A joint project between the New South Wales and Queensland Mining regulators in 2007 and 2008 resulted in the NSW document Managing Musculoskeletal Disorders A practical guide to preventing musculoskeletal disorders in the NSW mining and extractives industry as well as the Technical Report released by DEEDI Mines.

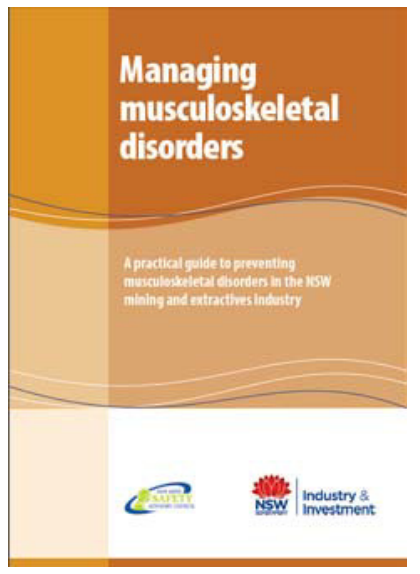

The guidance document was released in 2009, and has formed the basis of a number of musculoskeletal disorder prevention workshops in New South Wales, as well as the approach for the DEEDI Mines/Anglo Coal joint project.

\section{Barriers: Cultural issues with integration of ergonomics principles in Coal Mining}

The MSD risk management approach of identifying ergonomics hazards and eliminating or controlling those hazards is found in OHS legislation, $\mathrm{Na}-$ tional Code of Practice (2007), and the various state codes on manual handling or manual tasks. However, a report by Armour (2005) noted that, 'it would appear that much time and effort has gone into the in- jury management process without implementation of effective strategies to prevent future similar injury.'

The role of ergonomics hazards in mining equipment is well recognised; there are now many strategies in place to deal with these issues. Unfortunately, the consistent application of ergonomics risk management approaches in mining is competing with other messages-from conference presentations, general information available in the industry, or from consultants - that promote alternate approaches (for example the 'individual fitness' approach, or the manual handling training approach). Mining Safety and Health Conference presentations in recent years continue to have more focus on 'injury management', short term training or stretching programs, and functional capacity evaluations than on participatory ergonomics risk management initiatives. Some of this is due to issues with larger multinationals obtaining permission to share their projects and initiatives.

\section{Developing the industry benchmarks to promote Participatory Ergonomics principles in Coal Mining}

A small number of sites in Queensland Mining have undertaken a participatory ergonomics approach such as site specific ergonomics task forces. Two sites were visited during 2008 and 2009 that demonstrated different levels of commitment and interactions. Both these sites were featured as case studies in a Technical document. The key components of the programs were documented, and formed the basis of participatory ergonomics examples for Anglo Coal, Australia.

Rio Tinto Alcan Weipa 'translated' their Health Standards into site specific targets for reduction of key manual handling and ergonomic risks and form part of the annual site HSE continuous improvement plans. The key components of the program included a very stable workforce, a longstanding operation at the same site, and an experienced and interested health professional in the Safety Superintendent role. Higher level management support and a more developed health management strategy were also key factors.

At Crinum Mine in Central Queensland, the ergonomcs task force was facilitated by a consultant ergonomist, working with initially with equipment design. The key components at this site were the inter- 
est by one of the engineers in equipment design and ergonomics, and a culture of being an innovative site. As the task force had been in place a number of years, the issues with barriers to change had already been dealt with.

Further development of mining specific tools for risk assessment had been a focus of previous projects conducted by Robin Burgess-Limerick and associates, and funded by the Australian Coal Association Research Program (ACARP). The availability of risk assessment tools based on Manual Task risk factors in the Queensland government Manual Tasks Code of Practice (2010) also allowed the introduction of an approach that could be justified based on similar regulatory approaches. The key issue was selling the use of a Manual Task risk factor based approach to mining, rather than a generic risk assessment that had become the default approach.

\section{The progress and findings from the Anglo American Metallurgical Coal (AAMC) Participatory Ergonomics partnership}

The partnership plan has involved a series of meetings with key site personnel, with each site nominating a 'Champion' to coordinate the implementation. During 2011, site visits to the majority of AAMC sites allowed the AAMC Health Specialist (Project Leader) and the DEEDI Mines Ergonomist to gauge the level of acceptance from the senior management as well as from the workforce.

In August 2011, a participatory ergonomics 'train the trainer' session was run for site health personnel and contracted consultants providing physiotherapy services. As each site had (in conjunction with the DEEDI Ergonomist and the AAMC Health Specialist) discussed their current strategy, identified barriers, and also identified potential operational areas for prioritisation, the session was aimed at identifying gaps and developing realistic outcomes. AAMC had already determined that the key components of their internal strategy for MSD included:

- Resource the health roles at site level with personnel who have the appropriate professional skills to coordinate the sites efforts in MS injury prevention;
- Adopt the risk-based participatory ergonomics approach to MS injury prevention involving workers, health professionals and leaders at all levels;

- Improve the depth of investigations into MS injuries and scrutiny of the associated MS risks to ensure incident causation is clearly identified;

- Measure success by the demonstrated positive activities that have recognised and managed specific MS risks; and

- Record and share throughout AAMC successful MS injury risk controls developed by AAMC sites, identified in research findings, industry innovation, and known good practices.

In support for the cultural change component of the AAMC approach, the AAMC Project Leader has expressed that this is a 'life of mine' approach rather than an individual worker approach. Support for incorporating the components of the Queensland program into a corporate Ergonomics standard is being discussed.

\section{Benefits for the Queensland Mining Industry}

DEEDI Mines undertook the project in order to have an industry champion for the benefits of a 'Risk Management' approach to MSD prevention, involving participation of workers in solutions. A partnership with a major multinational mining company allows for sharing of good practice and plain English worker information that can be readily adopted by smaller companies with less resources. The project will result in a number of fact sheets and templates for Coal mines, and will assist with raising the profile of worker engagement in design of solutions.

\section{References}

[1] Armour, J. Effectiveness of Current Methods to control Sprain and Strain injuries in the Coal industry, Coal Health and Safety Trust, 2003

[2] Burgess-Limerick, R, Straker L, Pollock C, Dennis G, Leveritt $\mathrm{S}$, Johnson S , Implementation of the Participative Ergonomics for Manual Tasks (PErforM) Program at Four Australian Underground Coal Mines, International Journal of Industrial Ergonomics 2007, 37 (2): 145-155 